Nig. J. Biotech. Vol. 38 (1) : 154-159 (June 2021)

ISSN: 01891731

Available online at

NIGERIAN JOURNAL OF

BIOTECHNOLOGY

http://www.ajol.info/index.php/njb/index

and www.biotechsocietynigeria.org

DOI: https://dx.doi.org/10.4314/njb.v38i1.18

\title{
Enrichment of captive environment for effective animal reproduction
}

\author{
Oyeleye, 0.0 . \\ Animal Science Unit, Department of Agriculture, Wesley University Ondo.
}

\begin{abstract}
Animals are kept in captivity for the purpose of recreation, experimentation, rearing, reproduction, conservation, research etc. This study reviews how adequate welfare must be provided for the animals in captivity so that they will be able to perform to their maximum capabilities. It is imperative that the animals should not suffer because of the conditions they have been subjected to. The captive environment must be adequately enriched to foster effective reproduction that will sustain the population of the endangered species. This review has identified some of the problems faced by the captive animals and how to minimize these challenges. It is mandatory that before any animal is confined, adequate provision for its welfare must be provided to reduce any form of physiological or psychological stress.
\end{abstract}

Keywords: Captive animal, environmental enrichment, effective animal reproduction, animal welfare. Corresponding author: olubisi.oluseun@gmail.com/+2348160911765.

\section{Introduction}

Human population is expanding at a high rate and this calls for more land and resources. Wild animals and plants are being restricted to smaller areas in their natural habitat due to man's increasing demands for land for agricultural and developmental purposes. The populations of wild animals are being reduced and tend to extinction because their natural habitats are being destroyed. This has led to increase in captive population for some species while other species exist only in captivity. We have roughly 26 billion animals, covering over 10,000 species, being reared on farms, kept in zoos, conservation breeding centres, research laboratories and households (Beacham et al., 2001).

It is therefore important that the animal keepers develop the necessary skills, technologies and knowledge to maintain healthy life and breeding of the captive populations (Shepherdson et al.,
1998). The life of captive animals in zoos is affected by biotic and abiotic factors such as communal and spatial restrictions, the presence of other species including humans, and also the absence of the right environmentally induced stimuli for the development and exhibition of natural behaviours (Kleiman et al., 1996; Hosey, 2005).

Planning the management of captive wild mammals is a complex issue. Animal communication systems must be observed and considered when establishing an appropriate social environment and the information gained may aid managers in supervising the health and welfare of animals under their care (Kleiman et al., 1996; Snowdon, 1989). Ethology plays a vital role in understanding what animals suffer in different captive and wild environments and why they behave as they do. Ethology helps us to know the behaviour, preferences and wellbeing 
of animals both in the wild and captive environments. Kleiman et al. (1996) reveals that the manners by which wild animals behave led to creation of generations of natural selection and the adaptation to their niche. Captivity of wild animals can impose an environment vastly different from that in which they evolved. The ability of species to respond to their captive conditions depends on a complex interaction of developmental, experimental and genetic factors. Wild animals bred in captivity for relatively few generations have shown that the long term effect on behaviour is minimal, and to maintain captive breeding populations which exhibit behaviour as in the wild, conscious or intentional selection must be minimal (Altman, 1998).

Captive animals are often healthier, have a longer life span and reproduce more successfully than their wild con-specifics because they usually receive ample food and water, veterinary care and protection from predation and conflict (Mason, 2010; Kleiman et al., 1996).

On the other hand, captive environment is distinguished by their high population densities, limited spatial arrangement, low cannibalism, continuous availability of food and physical obstruction preventing dispersal and immigration (Newberry, 1995).

The study is aimed at how the captive environment can be enriched so that captive animals will be able to express their social behaviors which play important roles in their survival and effective reproduction in captivity.

\section{How captive environments affects the captive animals}

Animals in zoos, cages and encampments are referred to as captive animals because they are introduced from the wild to those places; the process of introduction from the wild is known as domestication. The captive environment affects the captive animals in three major ways: by induction of chronic stress, inability to control their surroundings and exhibition of stereotypic behaviour (Clare, and Romero, 2019).

Chronic stress. Animals show some reactions to captive environments by expressing chronic stress which seems to affect their physiological performance (Mason, 2010). Chronic stress in captive animals can present itself through the following physiological and psychological expressions of behaviours such as suppressed reproduction, increased abnormal behavior, increased self-injury, reduced exploratory behaviour, increased aggression, fearfulness etc. Inability to control their surroundings. One of the greatest problems experienced by captive animals is their inability to control their surroundings which has the following effects on them: they have no control over their social partners and mates, the amount of space between themselves and other conspecifics and between themselves and humans is small, what food they are offered and when offered (Morgan and Tromborg, 2007).

The third way by which the zoo environment affects captive animals is the exhibition of stereotypic behaviour which is caused by a brain dysfunction or frustration and is a repeated pattern with no apparent function or goal (Swaisgood and Shepherdson, 2005; Shepherdson et al., 1998; Jones \& Van Cantfort, 2007). When captive animals are deprived of their appropriate stimuli, they can express abnormal or repetitive behaviours. This stereotypic behaviour can present itself in different ways. Some animal species display a slight stereotypic behaviour because they have adjusted well to the captive environment, while other species show a profound amount of stereotypic behaviour (Sueur and Pelé, 2019).

How captive environments affect the reproductive behaviour and performance of the captive animals

A variety of environmental factors can influence the sexual behaviour of mammals, such as

Reduction in feed intake and inadequate nutrition. It has been proven that nutritional deficits can inhibit sexual development and interfere with physiological processes. McPherson and Chenoweth (2012) believed that providing safety, adequate nutrition and medical care will increase viability and reproductive success in comparison with free-living wild conspecifics. Promoting the welfare of the animals through better nutrition and integrating the activities of governments, professional bodies, scientists, extension workers and industries in supporting the implementation of good practices by the farmers themselves will enhance effective animal reproduction.

Absence of social groups: A captive environment does not maintain the animals in appropriate social groups which in turn prevents them from 
exhibiting some natural and wild-type behaviours (Farmer et al., 2011). For example, housing of small felids in groups larger than a pair leads to reduction in reproduction (Mellen, 1991).

Size and Quality of the Space: The size of a captive population is also limited by the space available in zoos and farms (Alexandre, 2009). The size and quality of the space are important in successful breeding and sometimes alterations in the physical environment can produce interesting changes in sexual behaviour. For example when female orangutans were given access to a second room where males could not follow, they showed higher levels of sexual solicitation (Kleiman et al., 1996).

Human interference: A captive environment is characterised by closeness to human interference. This condition can lead to manifestation of a range of behaviours due to disruption of the natural process. Human presence can interfere or hinder the expression of normal sexual behaviours. When such interference is absent, animals will be able to display appropriate phenotypes that will help them adapt to the captive condition and be able to have successful reproduction in captivity. The process of natural selection will be most intense during the first few generations after transition from wild to captive environments (Kleiman et al., 1996). Shepherdson et al (1998) discovered that the relationship between captive mammals and their keepers are extremely important, and that the animals depend on them for their security and wellbeing. This relationship if well managed will result into optimal reproductive success. A zookeeper who has related and responded well to animal responses to unsuitable conditions will make them reciprocate actions that will lead to reproductive success.

Female competition leading to reproduction suppression in subordinate females: Female competition for males is more widespread than acknowledged and involves a number of strategies, including the suppression of reproduction in subordinate females (McPherson and Chenoweth, 2012). Dominant females do so in subordinates at physiological and behavioural levels. This has been observed in several mammalian species that tend to be highly social and can have a well-developed dominant relationship (Creel et al., 1997).
Stress as a result of being captive born: Reproduction failure among captive born females may be due to the stress induced by the captivity they are subjected to. Swaisgood et al., (2006) reported that mated captive born females will not be able to produce offspring than wild caught females and this is because of some reproductive problems like post-copulation problem, inability to conceive or maintain pregnancy because of some wild or natural behaviours necessary for reproduction that are lacking or that they have failed to develop in the captive environment. A comparison of behaviour between wild and captive individuals is extremely useful in captive management and reintroduction programs (Swaisgood et al., 2006).

Physiological and behavioural problems can manifest in the forms of stress and stereotypes, and when captive animals are provided with enclosures that are not designed with any form of environmental enrichment, they can show signs of stress. This can affect their reproduction. Physiological problems faced by the captive animals are suppressed reproduction, increased abnormal behaviour, increased self-injury, reduced exploratory behaviour, and increased aggression and nervousness.

Species in captivity may face several problems which also include loss of genetic diversity, mutations, in-breeding, reduction in fitness and decrease or increase in reproduction (Fraser, 2008).

\section{Enhancing reproductive performance of captive animals}

Before animals in captivity can reproduce optimally, the following factors must be put in place:

Environmental enrichment: providing an environment that is similar to the wild to reduce inadvertent selection in captivity. This involves designing exhibits that can enhance the lifestyles of the captive animals, and it is within such naturalistic surroundings that typical patterns uf species will flourish e.g. large and free range exhibits, providing adequate animal welfare that will preserve their natural behaviours instead of changing their genetic adaptability, which will enhance successful 
breeding in captivity. Vegetative materials such as dirt, litter, mulch, vegetation, grasses or trees can enrich the environment and increase the biological complexity that are beneficial.

Improved healthcare: Giving adequate healthcare and management to captive animals can enhance their growth, development and reproductive performance. Adequate healthcare of the captive animals will ensure that they develop naturally as expected. This will boost their likelihood of survival especially when they are reintroduced into the wild.

Improved nutrition and better management: Giving adequate and nutritious feeds to captive animals can enhance their normal growth and reproductive performance. Provision of varieties of food can also enrich the environment (Newberry, 1993). The animals do not need to hunt for their survival. It is the responsibility of the animal keepers to provide their needs e.g. captive orange winged Amazon parrots (Amazona amazonica), when provided with environmental enrichment, were more productive in laying eggs (Hosey et al., 2005).

The use of assisted reproduction technologies: Captive animals that cannot reproduce themselves or have low survival percentages can be helped through the use of assisted reproductive technologies like Artificial Insemination (AI), Artificial Induced Spawning, Estrus Syncronisation, Multiple Ovulation and Embryo Transfer (MOET), In Vitro Maturation of oocytes (IVM), In Vitro Fertilisation (IVF), Surrogacy, Cryopreservation of semen, embryo, ovum etc. They can be kept under appropriate environmental conditions in which they can be assisted to reproduce artificially. Infertility of the wild infertile or subordinate female could be treated when isolated in the captive environment (Herrick, 2019; Oyeleye et al., 2016).

Keeping animals in appropriate social groups to stimulate natural and wild-type behaviours: Social partners will produce natural stimulation that will lead to effective reproduction without any medical assistance. Bored solely isolated animals are rejuvenated when appropriate partners are introduced or when kept in appropriate social groups. It allows them to relate more with their surroundings. Manipulating group size can also promote an increase in captive breeding (Farmer et al., 2011), for example housing small felids in groups larger than a pair leads to a reduction in reproduction (Mellen, 1991), whereas the presence of more than one female in black lemurs (Eulemur macaco) increases reproduction success (Hearn et al., 1996). Housing gorillas and other primates in large groups, a trend radically different from the past, has led to a great increase in successful breeding and natural rearing of the young (Kleiman et al., 1996; Hosey et al., 2005). Another example is that flamingos in the wild may form up to a million birds in a breeding season, but the smaller groups in zoos affect their breeding success (Stevens, 1991; Pickering et al., 1992). Stevens (1991) study of Caribbean flamingos (Phoenicopterus ruber) showed that group displays increased by $48 \%$ when the flock size was increased from 18 to $21 \%$ and synchronized by $100 \%$, there was also an increase in mounts, copulation and production of fertile eggs (the first in the group's history) (Stevens and Pickett, 1994). This example emphasizes the importance of providing a suitable con-specific social environment for captive animals (Hosey et al, 2005).

Reintroduction: Reintroduction is the release of captive born or wild caught animals into an area within their niche where their populations have declined or disappeared so that they will thrive and reproduce well to multiply the declining population for conservation or economic purposes. This is believed that it will improve the status of the wild population by increasing their numbers or changing their genetic makeup. This is appropriate when the species exists in small groups (Gadsby, 2002; Ware, 2001).

\section{Conclusion}

Captive animals need environments that are similar to their natural habitats for them to survive well. The captive environment must be enriched before the captive animals will be able to reproduce and fulfill every necessary condition for survival. The space allocation to the animals must be adequate to sustain a social group. The natural environment must be well simulated to support active life in the confinement. Adequate nutrition and veterinary care must be provided to solve the problems of hunting for food by the animals themselves. 
Variety of foods should be provided to reduce the effects of stereotypic behaviours imposed by the rearing environment. The animal husbandman must be ready to manage every form of interference which may pose a behaviour change or challenge to the animals in captivity. Effective reproduction is necessary to sustain the population of the captive animals, therefore it is important to adequately enrich the environment for successful performance.

\section{References}

Alexandre, R. (2009). Captive breeding genetics and reintroduction success. Biol. Conserv. 142, 29152922.

Altman, J. D. (1998). Animal activity and visitor learning at the zoo. Anthro. Zoos 11; 12-21.

Beacham, W., F.V. Castronova, B. Freedman, and S. Sessine (eds.). (2001). Beacham's Guide to Int. Endangered Spp. Gale Group, Farmington Hills, MI.

Clare, P. F. and Romero, M. L. (2019). Chronic captivity stress in wild animals is highly speciesspecific. Conserv. Physiol. 7:1.

Creel, S. Creel, N. Mills, M.G.L \& Monfort, S.L. (1997). Rank and reproduction in cooperatively breeding African wild dogs: behavioral and endocrine correlates. Behav. Eco., 8, 298306.

Farmer, H.L. Plowman, A.B. \& Leaver, L.A. (2011). Role of vocalizations and social housing in breeding in captive howler monkeys (Alouatta caraya). Appl. Ani. Behav. Sci., 134, 177-183.

Fraser, D. J. (2008). How well can captive breeding programs conserve biodiversity? A review of salmonids. Evol Appl. 1(4): 535-586.

Gadsby, L. (2002). Preparing for reintroduction: 10 years of planning for drills in Nigeria.

Reintroduction News, 2002:20-23.

Hearn, G.W. Berghaier, R.W \& George, D.D. (1996). Evidence of social enhancement of reproduction in two Eulemur species. Zoo Biol., $15,112$.

Herrick J. R. (2019). Assisted reproductive technologies for endangered species conservation: developing sophisticated protocols with limited access to animals with unique reproductive mechanisms. Biol. of Repro., Vol. 100:5,pp:150-

170,https://doi.org/10.1093/biolre/ioz025

Hosey, G.R. (2005). How does the zoo environment affect the behaviour of captive primates? Appl. Ani. Behav. Sci., 90, 107-129.

Jones, C.B. \& Van Cantfort, T.E. (2007). Multimodal communication by male mantled howler monkeys (Alouatta palliata) in sexual contexts: a descriptive analysis. Folia Primatologica, 78, 166-185.

Kleiman, D.G. Allen, M.E. Thompson, K.V. \& Lumpkin, S. (1996). Wild mammals in captivity. The University of Chicago Press, USA.

Mason, G.J. (2010). Species differences in responses to captivity: stress, welfare and the comparative method. Trends in Eco. \& Evo., 25, 20.

McPherson, F.J. \& Chenoweth, P.J. (2012). Mammalian sexual dimorphism. Ani. Reprod. Sci., 131, 109122.

Mellen, J.D., (1991). Factors influencing reproductive success in small captive exotic felids (Felis spp.): a multiple regression analysis. Zoo Biol., 10, 95-110.

Morgan, K. N., \& Tromborg, C. T. (2007). Sources of stress in captivity. Appl. Ani. Behav. Sci., 102(3-4), 262-

302. https://doi.org/10.1016/j.applanim.2006.05 .032 .

Newberry, R.C. (1995). Environmental enrichment: Increasing the biological relevance of captive environments. Appl. Ani. Behav. Sci., 44, 229243.

Oyeleye, O.O., Ola, S.I., Omitogun, O.G. (2016). Ovulation induced in African catfish (Clarias gariepinus, Burchell 1822) by hormones produced in the primary culture of pituitary cells. Int. J. Fish. Aquac. 8(7):67-73. 
Pickering, S. Creighton, E. and StevensWood, B. (1992). Flock size and breeding success in flamingos. Zoo Biol., 11, 229234.

Shepherdson, D.J. Mellen, J.D \& Hutchins, M. (1998). Second nature environmental enrichment for captive animals. Smithsonian institution press, Washington and London. ISBN: 1560983973.

Snowdon, C. T. 1989. The criteria for successful propagation of endangered species. Zoo Biol. ( Suppl . ) 1: 149-161.

Stevens, E.F. (1991). Flamingo breeding: the role of group displays. Zoo Biol., 10, 5363.

Stevens, E.F. \& Pickett, C. (1994). Managing the social environments of flamingos for reproductive success. Zoo Biol., 13, 501507.

Sueur C. \& Pelé M., (2019). "Importance of living environment for the welfare of captive animals: behaviours and enrichment" [PDF file], In: Hild S. \& Schweitzer L. (Eds), Ani. Wel.: From Sci. to Law, pp.175-188.

Swaisgood, R.R \& Shepherdson, D.J. (2005). Scientific approaches to enrichment and stereotypes in zoo animals: What's been done and where should we go next? Zoo Biol., 24, 499-518.

Swaisgood, R.R. Dickman, D.M \& White, A.M. (2006). A captive population in crisis: Testing hypotheses for reproductive failure in captive born southern white rhinoceros females. Biol. Conserv., 129, 468-476.

Ware, D. (2001). Gibbon rehabilitation and reintroduction: the problems along the road before use as a viable conservation tool. The Apes: Challenges for the 21st Century. Chicago, Brookfield Zoo, 259-261. 